\title{
Modeling Environmental Operative Elements in Agent-Based Pedestrian Simulation
}

\author{
Luca Crociani ${ }^{1}$, Giuseppe Vizzari ${ }^{1}$, Stefania Bandini ${ }^{1,2}$ \\ ${ }^{1}$ Complex Systems and Artificial Intelligence research center \\ University of Milano-Bicocca, Milano, Italy \\ \{name.surname\}@unimib.it \\ ${ }^{2}$ Research Center on Advanced Science and Technology, \\ The University of Tokyo, Tokyo, Japan
}

\begin{abstract}
Models for pedestrian simulation are employed on a day-to-day basis for supporting the design and planning of the built environment in normal and evacuation situations. One of the aspects that are least investigated in the community, probably because it is considered closer to technology transfer than to research, is the modelling of operational elements of the simulated environment. The present paper briefly describes an agent-based approach to the representation of operative elements of the environment with particular attention to the mechanisms of interaction between these active objects and pedestrians.
\end{abstract}

Keywords: agent-based simulation, environment, operative elements

\section{Modelling Operative Elements of Pedestrians' Environment}

Models for pedestrian simulation are employed on a day-to-day basis for supporting the design and planning of the built environment in normal and evacuation situations. Discrete modeling approaches are characterized by good computational properties (in particular the possibility to exploit parallel computing architectures) while preserving reasonable precision in the simulation of pedestrian dynamics. The Floor Field approach [1], originally proposed for Cellular Automata pedestrian models, represents a very general mechanism for managing operational level aspects of pedestrian dynamics in discrete modeling approaches. There are several extensions of the approach, for instance representing the effect of groups in the simulated pedestrian population [2][3], but also for the management of aspects that are difficult to incorporate within a discrete setting, like heterogeneous walking speeds [4][5], and particular shapes of the simulated environment (such as situations in which pedestrians perform circular movements [6]). It is important to notice that there are also extensions of this modeling approach that employ annotations to the discrete structure of the environment for the management of spatial information supporting tactical level decisions [7].

One of the aspects that are least investigated in the community, probably because it is considered closer to technology transfer than to research, is the modeling of operational elements of the simulated environment. We adopt this broad term to describe particular objects that are situated in the environment that is being modeled and simulated that influence the behavior of pedestrians by altering their velocity, due to the fact that they partly hinder a smooth movement, require pedestrians to perform some action to proceed, or that they purportedly limit overall flow, but that can also influence tactical level choices, for instance due to the fact that unbalanced queues can form as a consequence of their presence. A typical example of these objects is represented by turnstiles/ticket gates, but also resource distributors (e.g. ATMs). These objects are often crucial elements of the simulated scenarios, especially within the transportation area, and alternative numbers and arrangement of these elements is sometimes entirely motivating the simulation project. 


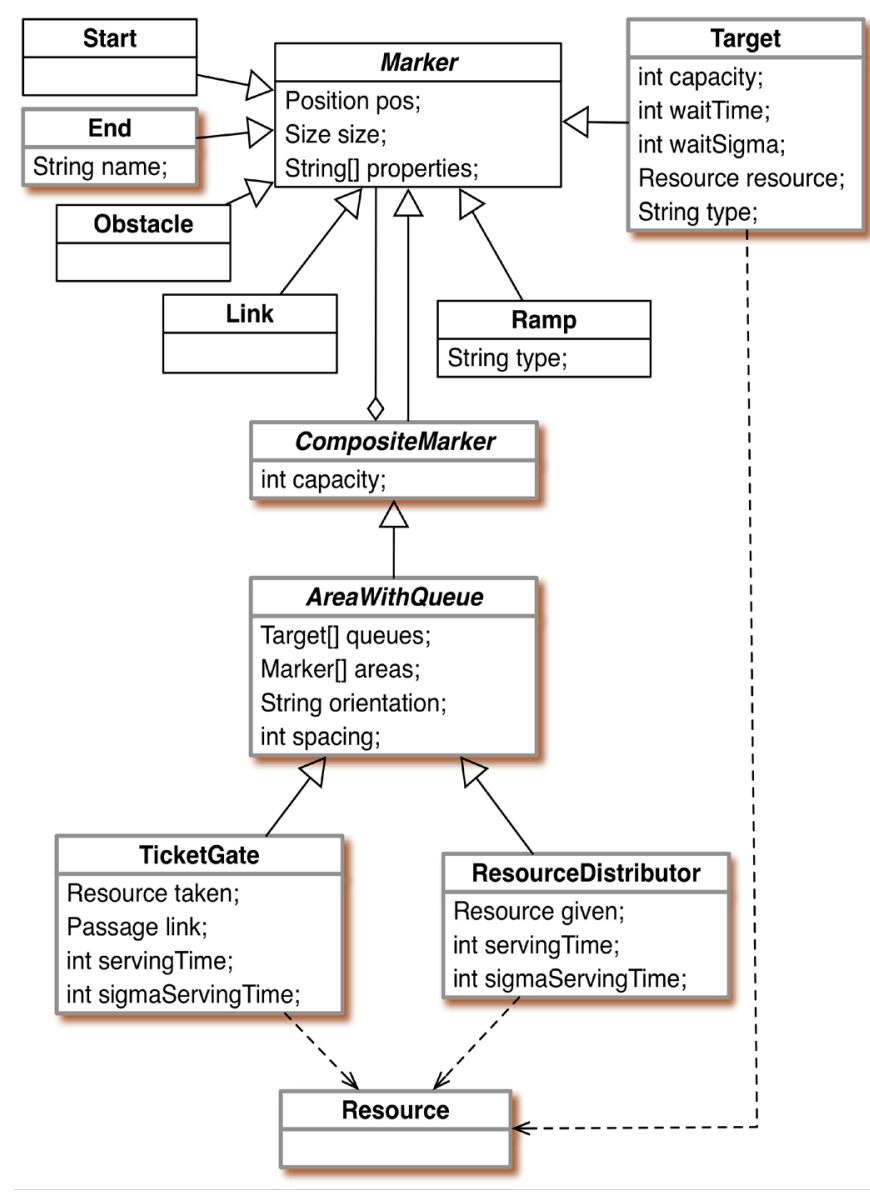

(a)

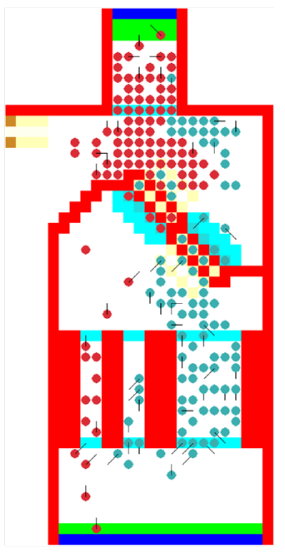

(b)

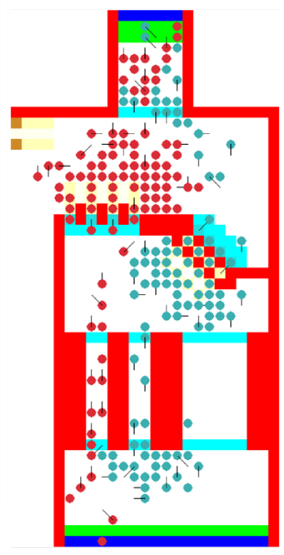

(d)

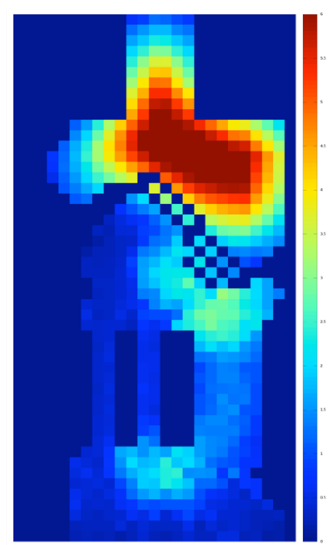

(c)

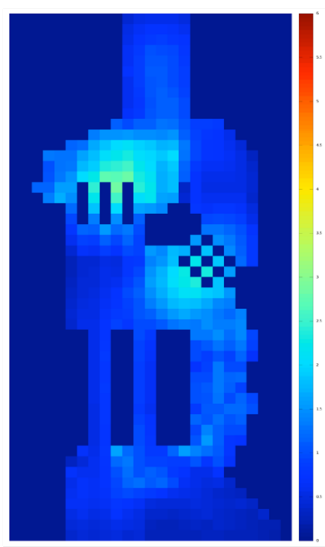

(e)

Figure 1- UML class diagram describing the organization of markers related to operative elements (a), and screenshots of alternative spatial arrangements (b and d) and cumulative mean densities ( $c$ and $e$ ).

The management of this kind of object within a pedestrian simulation models requires a more through consideration of the role of agents' environment [8][9], that is something more than a simple container of pedestrians, limiting their movements due to the presence of obstacles. The approach adopted for this work extends a model for operational and tactical level decisions (described in [7]) to represent and manage turnstiles and resource distributors. In particular, the environment in which agents are situated includes markers (their organization is shown in Figure 1a), that are particular sets of cells that play a role in supporting different aspects of agents' decisions and behaviors: these markers are in fact instrumental in the generation of floor fields, guiding pedestrian navigation at operational level, but they also compose higher level knowledge about the structure of the environment, owned by each agent. Within this context, some markers influence in an even deeper way agents' actions, in the vein of [10], leading them to form queues in front of distributors of objects that are necessary to carry out some specific actions (i.e. tickets to pass through a turnstile) or gates that require passing agents to provide an object (the above mentioned ticket) and that can also limit the overall flow of pedestrians, causing delays that can be calibrated to reflect real-world situations. These objects clearly influence both the tactical level decisions (i.e. "if I need to pass through a certain turnstile I need a ticket, so I have to go to a distributor first") as well as pushing agents to adopt some operational level alterations of their behavior (i.e. yield some turns of action to adapt to the 
maximum throughput flow associated to a certain turnstile), therefore cooperating with agents and performing a regulation function [11].

\section{Achieved Results}

Validation experiments based on "unit tests" (i.e. measuring the overall flow of pedestrians within a corridor with a single turnstile) have been performed also with the aim of actually calibrating some prameters of objects like turnstiles, also with the aim of supporting forms of verification tests as suggested by [12]. We also considered a more complicated example scenario, presenting most typical elements of a transport station but in a much smaller scale, to support a comparison among alternative arrangements for the same number of operative elements within a small entrance to a transit station, as shown in Figure $1 \mathrm{~b}$-Figure 1e. The effect of different position and arrangement of turnstiles has a significant effect on spatial utilization patterns, depicted in terms of cumulative mean density, qualitatively showing how this kind of object could be relevant to generate what-if scenarios fully exploiting pedestrian simulation models.

\section{Conclusions and Future Works}

The adopted approach represents a way to model and implement operative elements of pedestrians' environment within an agent-based framework, and it also represents a starting point for modelling additional and potentially more complicated elements, such as elevators: the latter would require the definition of additional mechanisms for the operative element per se (in particular for the specification of the way the elevator responds to requests from different floors), but also potentially additional behavioural patterns for pedestrians (for instance to manage the social norms governing the process of exiting and boarding).

\section{References}

[1] C. Burstedde, K. Klauck, A. Schadschneider, and J. Zittartz, "Simulation of pedestrian dynamics using a two-dimensional cellular automaton," Phys. A Stat. Mech. its Appl., vol. 295, no. 3-4, pp. 507-525, Jun. 2001.

[2] G. Vizzari, L. Manenti, and L. Crociani, "Adaptive Pedestrian Behaviour for the Preservation of Group Cohesion," Complex Adapt. Syst. Model., vol. 1, no. 7, 2013.

[3] F. Müller, O. Wohak, and A. Schadschneider, "Study of Influence of Groups on Evacuation Dynamics Using a Cellular Automaton Model," Transp. Res. Procedia, vol. 2, pp. 168-176, Jan. 2014.

[4] A. Kirchner, H. Klüpfel, K. Nishinari, A. Schadschneider, and M. Schreckenberg, "Discretization effects and the influence of walking speed in cellular automata models for pedestrian dynamics," J. Stat. Mech. Theory Exp., vol. 2004, no. 10, p. P10011, Oct. 2004.

[5] S. Bandini, L. Crociani, and G. Vizzari, "An approach for managing heterogeneous speed profiles in cellular automata pedestrian models," J. Cell. Autom., vol. 12, no. 5, 2017.

[6] K. Shimura, S. D. Khan, S. Bandini, and K. Nishinari, "Simulation and evaluation of spiral movement of pedestrians: Towards the tawaf simulator," J. Cell. Autom., vol. 11, no. 4, pp. 275284, 2016.

[7] L. Crociani, A. Piazzoni, G. Vizzari, and S. Bandini, "When reactive agents are not enough: Tactical level decisions in pedestrian simulation," Intelligenza Artif., vol. 9, no. 2, pp. 163-177, Dec. 2015.

[8] D. Weyns, A. Omicini, and J. Odell, "Environment as a First Class Abstraction in Multiagent Systems," Auton. Agents Multi-Agent Syst., vol. 14, no. 1, pp. 5-30, 2007.

[9] A. Helleboogh, G. Vizzari, A. Uhrmacher, and F. Michel, "Modeling dynamic environments in multi-agent simulation," Auton. Agent. Multi. Agent. Syst., vol. 14, no. 1, pp. 87-116, 2007.

[10] S. Paris and S. Donikian, "Activity-Driven Populace: A Cognitive Approach to Crowd Simulation," IEEE Comput. Graph. Appl., vol. 29, no. 4, pp. 34-43, 2009.

[11] S. Bandini and G. Vizzari, "Regulation Function of the Environment in Agent-Based Simulation," in Environments for Multi-Agent Systems III, Third International Workshop, E4MAS 2006, Hakodate, Japan, May 8, 2006, Selected Revised and Invited Papers, 2007, vol. 4389, pp. 157- 
169.

[12] E. Ronchi, E. D. Kuligowski, P. A. Reneke, R. D. Peacock, and D. Nilsson, "The Process of Verification and Validation of Building Fire Evacuation Models," Gaithersburg, MD, Nov. 2013. 